\title{
In vitro processing of glutamyl endopeptidase proenzymes from Enterococcus faecalis and importance of $\mathbf{N}$-terminal residue in enzyme catalysis
}

\author{
Shakh M. A. Rouf ${ }^{1,2, ~ *, ~ Y . ~ O h a r a-N e m o t o ~}{ }^{1}$, T. Ono ${ }^{1}$, Y. Shimoyama ${ }^{3}$, S. Kimura ${ }^{3}$, T. K. Nemoto ${ }^{1}$ \\ ${ }^{1}$ Department of Oral Molecular Biology, Courses of Medical and Dental Science, Nagasaki University Graduate School of Biomedical \\ Sciences, Nagasaki 852-8588, Japan \\ ${ }^{2}$ Department of Applied Nutrition \& Food Technology, Islamic University, Kushtia-7003, Bangladesh \\ ${ }^{3}$ Division of Molecular Microbiology, Iwate Medical University, Yahaba-cho 028-3694, Japan
}

\section{Email address:}

abdurrouf_7@yahoo.com (S. M. A. Rouf)

\section{To cite this article:}

Shakh M. A. Rouf, Y. Ohara-Nemoto, T. Ono, Y. Shimoyama, S. Kimura, T. K. Nemoto. In Vitro Processing of Glutamyl Endopeptidase Proenzymes from Enterococcus faecalis and Importance of N-terminal Residue in Enzyme Catalysis. Advances in Biochemistry.

Vol. 1, No. 5, 2013, pp. 73-80. doi: 10.11648/j.ab.20130105.11

\begin{abstract}
Glutamyl endopeptidase from Enterococcus faecalis, designated SprE, is one of the important virulence factors secreted as zymogen. In the present study we expressed recombinant SprE proenzyme (pro-SprE) in Escherichia coli and investigated the in vitro processing to mature SprE. It was found that trypsin could efficiently produce the active form of SprE with the N-terminus $\operatorname{Ser}^{1}$ through cleavage between $\operatorname{Arg}^{-1}$ and $\operatorname{Ser}^{1}$ bond, which was subsequently auto-degraded into inactive species through the cleavage at the $\mathrm{Glu}^{6}-\mathrm{Asp}^{7}$ and $\mathrm{Glu}^{11}-\mathrm{Val}^{12}$ bonds. Although thermolysin could produce SprE with the $\mathrm{N}$-terminus Leu ${ }^{2}$, but possessed no proteolytic activity. In contrast to the absolute requirement of the N-terminal Val ${ }^{1}$ in staphylococcal glutamyl endopeptidases, the N-terminal Ser $^{1}$ of mature SprE could be substituted by other amino acids despite that Ser showed the maximal activity. Substitution of penultimate $\mathrm{Leu}^{2}$ of SprE to $\mathrm{Val}^{2}$ also reduced the activity to $40 \%$ of the wild type. Taken together, we conclude that pro-SprE was converted to mature form with the N-terminus $\operatorname{Ser}^{1}$ by a protease with specificity of trypsin and the length of the N-terminal region rather than specific residue is absolutely required for enzyme activity.
\end{abstract}

Keywords: Glutamyl Endopeptidase, Proenzyme Processing, Spre, Enterococcus faecalis, Gluv8

\section{Introduction}

Enterococcus faecalis is a gram-positive cocci and common inhabitant of human gastrointestinal and genitourinary tract [1]. It causes a wide variety of diseases in human, infecting the urinary tract, endocardium, abdomen, biliary tract, burn wounds and indwelling foreign devices [2]. In dentistry, E. faecalis has been associated with caries lesions, chronic periodontitis, recurrent root canal infection and persistent apical periodontitis [3, 4]. Candidate virulence factors of $E$. faecalis include: cytolysin and proteolytic enzyme (gelatinase and serine protease), adhesions (aggregation substance, enterococci surface protein or Esp, collagen adhesion protein or Ace, antigen A or EfaA) and capsular and cellular wall polysaccharides [2, 5]. Serine protease, gelatinase and collagen-binding protein (Ace) facilitate $E$. faecalis bind to dentin [6]. Several studies have been undertaken to investigate the possible role of gelatinase and serine proteases in the disease processes caused by E. faecalis and they were found to contribute to the pathogenesis through biofilm formation, facilitating bacterial invasions, degradation of immune peptides required for host response [7, 8, 9,10]. Gelatinase (GelE) and serine protease (SprE) expressed in E. faecalis were reported to be important for enterococcal virulence in mouse peritonitis model [11]. Previous studies also indicated that the presence of extracellular proteases, GelE and SprE, in the E. faecalis raised mortality in animal models [12, 13].

Glutamyl endopeptidase (EC 3.4.21.19) from Staphylococcus aureus V8 strain (GluV8) is a serine protease with unique substrate specificity to Glu-X and Asp-X with the higher preference to the former [14,15]. This family protease from $E$. faecalis $\mathrm{SprE}$, which has been shown to contribute to pathogenesis in animal models [16, $11,17]$. Kawalec et al. [18] purified several isoforms of 
mature SprE, starting with N-terminal Ser ${ }^{1}$ and $\mathrm{Leu}^{2}$ from wild-type E. faecalis OG1RF (TX4002) and a gelatinase-null mutant TX5264, among which SrpE starting with Ser $^{1}$ was super active. Moreover, they suspected the instability of active SprE ( $\operatorname{Ser}^{1}-\mathrm{SprE}$ form) apparently due to auto-degradation.

SprE shows sequence homology with GluV8 (27\% identity, 49\% similarity) [19] and that from $S$. epidermidis (GluSE) (26\% identity, 49\% similarity) [20]. It has been reported that the proenzyme of GluV8 is processed by a thermolysin-family metalloprotease, aureolysin in vivo $[21,22]$. The prosequence of Staphylococcal GluV8-family protease was reported to sequentially remove to shorter proenzymes in an autocatalytic manner by cleaving at Glu-X and Gln-X in the prosegment and finally matured by aureolysin at N-terminal $\mathrm{Asn}^{-1}-\mathrm{Val}^{1}$ [23, 24]. Consistently recombinant GluV8 expressed in E. coli was efficiently processed to mature GluV8 by thermolysin in vitro [25]. $\mathrm{Val}^{1}$ is conserved at the N-termini of mature glutamyl endopeptidases from Staphylococcus epidermidis [26, 27], Staphylococcal warneri [28], Staphylococcal cohnii and Staphylococcal caprae [29].

The N-terminus amino acid residue of mature GluV8-family endopeptidases was found to be critical for maturation by aureolysin and for the stabilization of mature structure resistant to further processing at the $\mathrm{Val}^{1}-\mathrm{Ile}^{2}$ and $\mathrm{Ile}^{2}-\mathrm{Leu}^{3}$ bonds [25]. Furthermore, $\mathrm{Val}^{1}$ is directly involved in the protease activity itself, because $\mathrm{Val}^{1}$ cannot be substituted by other amino acids, even if the processing was correctly mediated with $\mathrm{Arg}^{-1}-\mathrm{X}^{1}$-substituted forms by trypsin (X=Ala, Phe, Gly and Ser) [25]. Exceptionally, the $\mathrm{Val}^{1}$ substitution to Leu ${ }^{1}$ could partially $(30 \%)$ retained proteolytic activity of the wild type, further indicating the significance of $\mathrm{Val}^{1}$ for the protease activity [29]. Sequence alignment indicates that Val ${ }^{1}$ of GluV8 corresponds to Leu ${ }^{2}$ of SprE, whereas it has been reported that SprE with N-terminal Leu $^{2}\left(\mathrm{Leu}^{2}\right.$-SprE) had a significantly negligible activity compared to that with N-terminal $\operatorname{Ser}^{1}$ [18]. Moreover, the processing mechanism of pro-SprE still remains unknown. Therefore, we here investigated the in vitro processing of pro-SprE expressed in $E$ coli and addressed the roles of $\mathrm{N}$-terminal amino acids in the enzyme catalysis.

\section{Materials and Methods}

\subsection{Materials}

The materials used and their sources were as follow: expression vector pQE60, from Qiagen Inc. (Chatsworth, CA, USA); low-molecular-weight markers, from GE Healthcare (Buchinghamshire, England); restriction enzymes and DNA-modifying enzymes, from Nippon Gene (Tokyo, Japan); KOD Plus DNA polymerase, from Toyobo (Tokyo, Japan); Talon metal-affinity resin, from Clontech Laboratories Inc. (Palo Alto, CA, USA); Z-Leu-Leu-Glu-MCA (LLE-MCA),
Ac-Thr-Val-Ala-Asp-MCA

Z-Ala-Ala-Asn-MCA

(AAN-MCA)

(TVAD-MCA), Boc-Gln-Ala-Arg-MCA (QAR-MCA)from the Peptide Institute Inc. (Osaka, Japan);Leu-Asp-MCA (LD-MCA) and Z-Leu-Leu-Gln-MCA (LLQ-MCA), synthesized by Thermo Fisher Scientific (Ulm, Germany); thermolysin from Bacillus thermoproteolyticusrokko, trypsin procine pancreas, bovine serum albumin (BSA), were from Sigma-Aldrich (St. Louis, MO, USA); and gelatin from the NacalaiTesque. Inc. (Osaka, Japan).

\subsection{Expression Vector for the Expression of SprE}

The SprE gene was amplified by PCR using KOD Plus DNA polymerase and chromosomal DNA $(0.1 \mu \mathrm{g})$ of $E$. faecalis NCTC 775 as template. The synthetic oligonucleotides primers (5'-ATGGATCCAAAAAGTTCTCCATACGAAAAATTA G-3') and (5'-GTGGATCCCGCTGCAGGCACAGCGGATAAACG3') containing BamHI sites (underlined) were designed on the basis of the DNA sequence of SprE (GeneBank accession no. Z12296), used for SprE gene amplification. PCR-amplified 0.8-kb fragment (without stop codon) was cleaved with BamHI, and then inserted into the BamHI site of pQE60 to yield pQE60-SprE expression vector.

\subsection{Expression and Purification of Recombinant Proteases}

In order to minimize the modification in the N-terminal preprosequence of SprE, the expression vector pQE60 that encoded an affinity tag, [Gly-Ser-Arg-Ser-(His) 6 ] at the C-terminus was used (Fig. 1A). In addition Gly-Gly-Ser derived from the vector was present between 1st-Met and $2^{\text {nd }}$-Lys of the N-terminal prepropeptide. Escherichia coli XL1-blue was transformed with the pQE60-SprE expression plasmids. C-terminal $\mathrm{His}_{6}$-tagged recombinant proteins were expressed and purified as described previously [25].Protein concentrations were determined by the bicinchoninic acid method using BSA as the standard (Pierce, Rockford, IL, USA). Purified proteins were stored at $-80{ }^{\circ} \mathrm{C}$ until used.

\subsection{Amino Acid Numbering and In Vitro Mutagenesis}

The N-terminal Ser of the active SprE was numbered as the first amino acid residue (Ser1). In vitro mutagenesis was performed as reported previously [30] by PCR with mutated primer(s) to substitute 3 amino acids in the prosequence (Glu ${ }^{-15}$ Ser, Glu ${ }^{-14}$ Lys, Glu ${ }^{-8}$ Ile, designated as SprE-mut), 4 amino acids in the mature region (Glu11Gln, Glu6Gln, Ser1Thr/Ala/Val and Leu2Val), and an essential Ser ${ }^{180}$ to Ala. All mutations were confirmed by DNA sequencing.

\subsection{SDS-PAGE and Zymography}

Recombinant proteins (1 $\mu \mathrm{g})$ were separated by SDS-PAGE at a polyacrylamide concentration of $12.5 \%$ $(\mathrm{w} / \mathrm{v})$, and then stained with Coomassie Brilliant Blue 
(CBB). Band intensities wer quantified with Image $J$ software [31]. Zymography was performed by using a polyacrylamide gel containing $1 \mathrm{mg} / \mathrm{ml}$ of gelatin (instead of azocasein) according to the method previously described [32]. For zymography, thermolysin- or trypsin-treated samples $(0.25 \mu \mathrm{g})$ were separated on a $12.5 \%$ polyacrylamide gel containing $1 \%(\mathrm{w} / \mathrm{v})$ of gelatin by SDS-PAGE, the gel was incubated twice with $100 \mathrm{ml}$ of $2.5 \%$ (v/v) of Triton X100 at $25{ }^{\circ} \mathrm{C}$ for $20 \mathrm{~min}$, twice for $10 \mathrm{~min}$ each time with $100 \mathrm{ml}$ of 50 mMTris- $\mathrm{HCl}(\mathrm{pH} 7.8)$ containing $30 \mathrm{mMNaCl}$, and then incubated overnight at 37 ${ }^{\circ} \mathrm{C}$ in $100 \mathrm{ml}$ of the new batch of the same buffer containing $0.001 \% \mathrm{NaN}_{3}$. Finally, non-hydrolyzed gelatin in the gel was visualized by CBB staining.

\subsection{In Vitro Processing of Recombinant Proteases and the Measurement of the Protease Activity}

Recombinant proteins $(10 \mu \mathrm{g})$ were incubated for $2 \mathrm{~h}$ in $0.1 \mathrm{ml}$ of buffer A [10 mM sodium borate $(\mathrm{pH} 8.0)$ containing $0.005 \%(\mathrm{v} / \mathrm{v})$ Triton $\mathrm{X} 100$, and $2 \mathrm{mM} \mathrm{CaSO}_{4}$ ] without or with $0.3 \mu \mathrm{g}$ of thermolysin (molar ratio of 33:1) and $0.003 \mu \mathrm{g}$ of trypsin (molar ratio $3300: 1$ ) at $37^{\circ} \mathrm{C}$, unless otherwise stated. Thereafter, proteins $(0.25 \mu \mathrm{g}$ as proform)were incubated at $37^{\circ} \mathrm{C}$ for $1 \mathrm{~h}$ with $20 \mu \mathrm{M}$ MCA peptides in $0.2 \mathrm{ml}$ of $50 \mathrm{mMTris}-\mathrm{HCl}(\mathrm{pH} 8.0)$ containing 5 mM EDTA. The fluorescence was measured with excitation at $380 \mathrm{~nm}$ and emission at $460 \mathrm{~nm}$ with a Fluorescence Photometer F-4000 (Hitachi, Tokyo, Japan).

\subsection{N-terminal Amino Acid Sequencing}

$\mathrm{N}$-terminal amino acid sequences of recombinant SprE and its derivatives were determined after separation by SDS-PAGE $(2 \mu \mathrm{g})$ and transference to a polyvinylidenedifluoride membrane (Sequi-Blot PVDF Membrane, Bio-Rad, Hercules, CA, USA). After having been stained with $\mathrm{CBB}$, the bands were excised; and directly sequenced with a Procise $49 \mathrm{XcLC}$ protein sequencer (ABI, Foster City, CA, USA).

\section{Results}

\subsection{Expression and In Vitro Processing of SprE}

When the full-length pro-SprE was expressed in E. coli, a mixture of $34-$ and $35-\mathrm{kDa}$ proteins was recovered by affinity purification (Fig. 1a, upper panel, lane 1). Amino acid sequencing revealed that the $35-\mathrm{kDa}$ band started at $\mathrm{Glu}^{-14}$ and $34-\mathrm{kDa}$ one at $\mathrm{Ser}^{-7}$, indicating the autocatalytic prosequence cleavage between the $\mathrm{Glu}^{-15}-\mathrm{Glu}^{-14}$ and $\mathrm{Glu}^{-8}-\mathrm{Ser}^{-7}$ bonds, respectively (Table 1).The 34-35-kDa SprE showed negligible glutamic acid-specific peptidase activity, as recombinant SprE still carried part of the propeptide (Fig.1b,c upper; d). Staphylococcal GluV8-family proteases were reported to be processed into mature form by thermolysin cleaving at $\mathrm{Asn}^{-1}-\mathrm{Val}^{1}$ in vitro [29]. To investigate heterocatalytic maturation of pro-SprE, we incubated 34-35-kDa recombinant pro-SprE with trypsin or thermolysin, expected that may induce processing at $\mathrm{Arg}^{-1}-\mathrm{Ser}^{1}$ and at $\mathrm{Ser}^{1}-\mathrm{Leu}^{2}$ sites respectively. Consequently, trypsin treatment accompanying a slight decrease in the apparent molecular mass induced an acquisition of the gelatinase activity (Fig. 1c, lanes 3-10). In contrast, thermolysin treatment did not accompany an apparent increased gelatinase activity, although the apparent molecular mass was decreased. Very faint gelatinase activities were found for thermolysin treated or untreated samples (Fig.1b lower). In consistent to these findings, the Glu-specific peptidase activity was measured for the trypsin treatad Corf in a dnos danandant mannar (Fir $1 \mathrm{~d}$ )
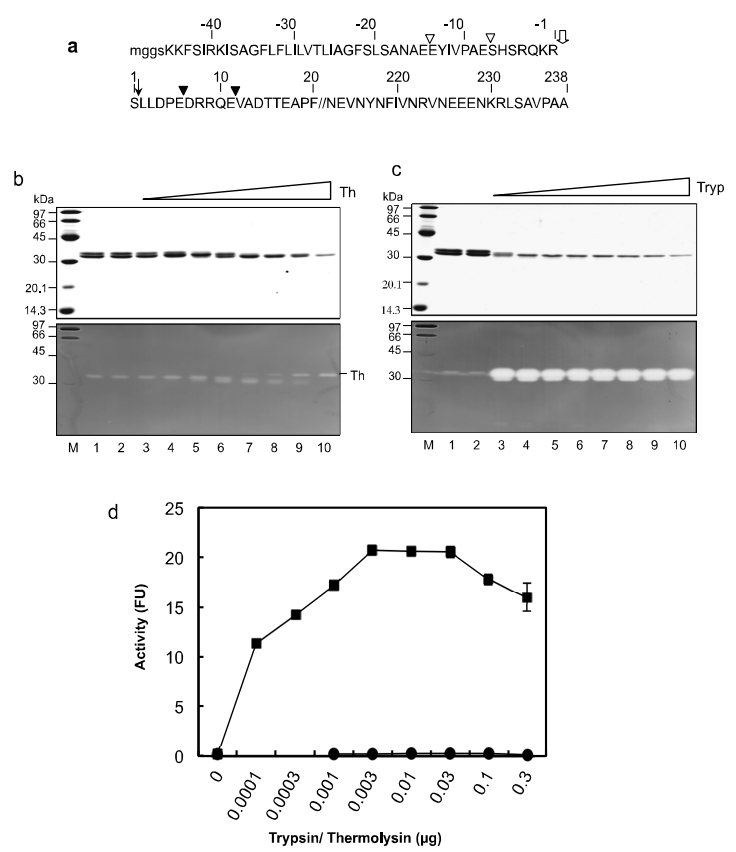

Fig 1. A schematic presentation of pro-SprE expressed in E. coli. (a) Amino acid sequences of Pre-pro and $N$-terminal regions of mature SprE. The open $(\nabla)$ and closed $(\boldsymbol{\nabla})$ arrow-head showing the trypsin (Tryp) and thermolysin (Th) processing site respectively, arrow $(\downarrow)$ indicates the site of autodegradation in the pro-and mature region. (b,c) In vitro processing of the SprE proenzymes and Z-LLE-MCA cleavage activity of the mature

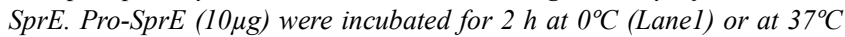
(lane2) without Thermolysin (Th)/Trypsin (Tryp) and with Th-

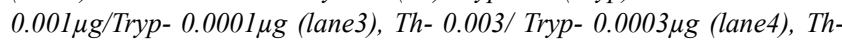

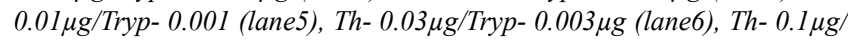

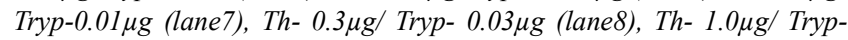

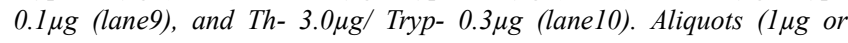
$0.25 \mu \mathrm{g}$ ) of each Thermolysin/ Trypsin treated samples were subjected to SDS-PAGE and then stained with $C B B$ (b,c upper) or subjected to collagen-Zymography (b,c lower) respectively. (d) The proteolytic activities (means $\pm S D ; n=3$ ) towards Z-LLE-MCA of the thermolysin (Th) and trypsin (Tryp) treated samples were determined as described in the materials and methods.

$\mathrm{N}$-terminal sequencing of thermolysin-processed $31-\mathrm{kDa}$ species revealed a cleavage at the $\mathrm{Ser}^{1}-\mathrm{Leu}^{2}$ bond, whereas the N-terminus of trypsin-processed species was a mixture of SprE with N-terminus Val ${ }^{12}$ (major) and $\operatorname{Ser}^{1}$ (minor) (Table 1). Since the Glu ${ }^{11}-\mathrm{Val}^{12}$ bond should not be processed by trypsin, but by glutamyl endopeptidase itself, it was reasonably suggested that mature SprE with N-terminal 
$\operatorname{Ser}^{1}\left(\operatorname{Ser}^{1}-\mathrm{SprE}\right)$ produced by trypsin readily auto-degraded to the $\mathrm{Val}^{12}$-SprE. However, the possibility that $\mathrm{Val}^{12}-\mathrm{SprE}$ represents a genuine mature species with the peptidase activity was not completely eliminated, if considered that N-terminal Val ${ }^{1}$ was commonly observed in Staphylococcal mature glutamyl endopeptidases [29]. To address these possibilities, we tried to increase recovery of Ser ${ }^{1}$-SprEquantitatively after trypsin processing by introducing amino acid substitutions in the N-terminal region of the mature SprE as in the following section.

\subsection{Suppression of Auto-Degradation of Mature SprE}

The autodegradation of Staphylococcal glutamyl endopeptidases occurring within the proseuqence region was efficiently suppressed by the substitution of Glu and Asp in the proseqquences, to Gln, Asn or other amino acids $[25,30]$. Here, this strategy was introduced at the N-terminal region of mature SprE.

When $\mathrm{Glu}^{11}$ of SprE was substituted to Gln (SprE-Glu11Gln), the 31-kDa mature SprE band was more intensively recovered after trypsin treatment of the purified proenzyme (Fig-2a upper lane6), which accompanied by an increased peptidase activity in the fraction (Fig-2a lower).
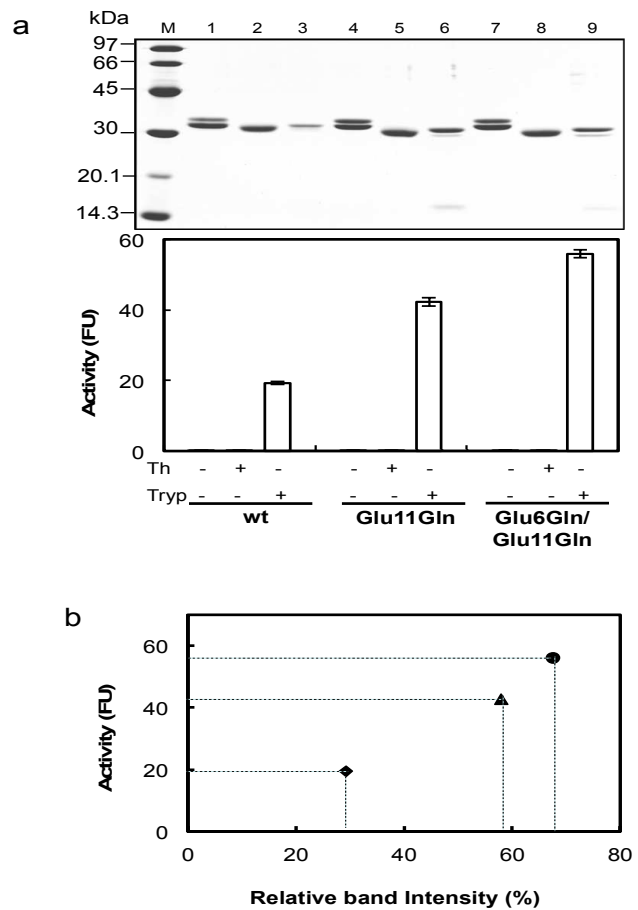

Fig 2. Prevention of auto-degradation and recovery enhancement of

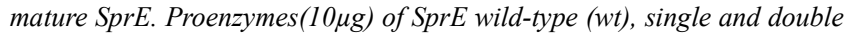
amino acid substitution mutants Glu6Gln and Glu6Gln/GlullGln respectively were incubated for $2 \mathrm{~h}$ at $37^{\circ} \mathrm{C}$ without thermolysin (Th)/

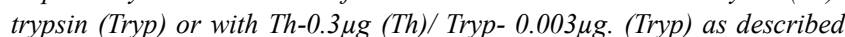
in the materials and methods section. (a) Proteins $(1 \mu \mathrm{g})$ were separated by $S D S-P A G E$, stained with $C B B$ and proteolytic activities (mean $\pm S D ; n=3$ ) of the samples were determined with LLE-MCA as per materials and methods section. (b) The band intensities of the trypsin treated mature

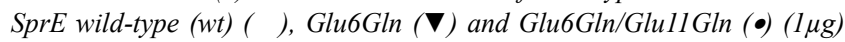
were determined by using image $J$ software [31]. Band intensities of pro-SprE set as $100 \%$.
Because there was $\mathrm{Glu}^{6}$ at position nearer to the N-terminus, this residue was also substituted by Gln (SprE-Glu6Gln/Glu11Gln). As a result, the recovery was further increased after trypsin treatment (Fig-2a upper lane9). It is noticeable that the specific activity of the mutant form was not varied (Fig-2b), whereas the recovery after the processing was increased by suppression of the auto-degradation. Even when thermolysin treatment was conducted on these mutants, no increase in recovery (Fig-2a upper lane $5 \& 8$ ) as well as in the activity (Fig-2a lower panel) was observed again. The N-terminus of trypsin-processed SprE-Glu11Gln was a mixture of $\operatorname{Ser}^{1}$ and $\mathrm{Asp}^{7}$ and that of SprE-Glu6Gln/Glu11Gln was Ser ${ }^{1}$. Taken together with the activity measurement, it was concluded that $\operatorname{Ser}^{1}-\mathrm{SprE}$ was the active and mature form and that $\mathrm{Val}^{12}$-SprE as well as Asp - SprE was an inactive auto-degraded product (Table 1).

\subsection{Substrate Specificity of Pro- and Mature SprE}

Glutamyl endopeptidase cleaved peptide bond between Glu-X with highest efficiency and much less efficiently between Asp-X [15]. We investigated the substrate specificity of pro- and mature SprE with several peptidyl-MCA substrates to know whether any possibility of propeptide processing in an autocatalytic manner. Mature Ser ${ }^{1}$-SprE specifically hydrolyzed LLE-MCA, and did not hydrolyze TVAD-, LD-, AAN-MCA as well as GAR-MCA, whereas it possessed very faint activity for LLQ-MCA after $1 \mathrm{~h}$ incubation (Fig-3).

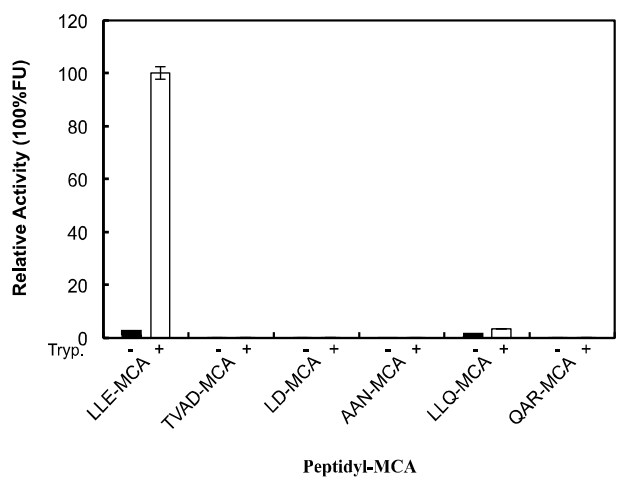

Fig 3. Substrate specificity of SprE. Substrate specificity of pro- and mature SprE was determined with different MCA-peptides as shown in the figure bellow. Proteolytic activity towards Z-LLE-MCA of trypsin processed mature SprEwt set as positive control and considered $100 \%$. Pro-SprE (10 $\mu \mathrm{g})$ was incubated for $2 \mathrm{~h}$ at $37^{\circ} \mathrm{C}$ without or with $0.003 \mu \mathrm{g}$ trypsin (molar ratio 3300:1), there after $0.25 \mu \mathrm{g}$ of each sample was used for different MCA-peptides proteolytic assay as discussed in the materials and methods. 
Table 1. N-terminal amino acid sequences of recombinant SprE and its derivatives

\begin{tabular}{|c|c|c|}
\hline SprE derivatives & Thermolysin (Th)/Trypsin (Tryp) & Cleavage site (X-/-X) \\
\hline pro-SprE & $-a^{a}$ & $\mathrm{E}^{-15} / \underline{\mathrm{E}}^{-14} \mathrm{YIVPAE}^{-8} / \underline{\mathrm{S}^{-7} \mathrm{RQKRSLLDPED}}$ \\
\hline \multirow[t]{2}{*}{ pro-SprEmut } & - & $\mathrm{SHSQ}^{-3} / \underline{\mathrm{K}^{-2} \mathrm{RSLLDPEDRRQ}}$ \\
\hline & Th & SHSQKRS ${ }^{1} / \underline{L}^{2}$ LDPEDRRQ \\
\hline \multirow[t]{2}{*}{ SprEwt } & $\operatorname{Tryp}^{b}$ & $\mathrm{SHSQKR}^{-1} / \underline{\mathrm{S}^{1} \text { LLPEDRRQ }}$ \\
\hline & & $\mathrm{RRQE}^{11} / \underline{\mathrm{V}^{12} \mathrm{ADTTE}} \mathrm{A}$ \\
\hline \multirow[t]{3}{*}{ SprE-Glu11Gln } & Th & $\mathrm{KRS}^{1} / \mathrm{L}^{2} \mathrm{LDPEDRRQ}$ \\
\hline & Tryp $^{c}$ & $\mathrm{SHSQKR}^{-1} / \underline{\mathrm{S}^{1} \text { LLDPEDRRQ }}$ \\
\hline & & $\mathrm{KRSLLDPE}^{6} / \underline{\mathrm{D}}^{7} \mathrm{RRQQVADT}$ \\
\hline SprE-Glu6Gln/Glu11Gln & Tryp & SHSQKR $^{-1} / \underline{\mathrm{S}^{1} \text { LLDPQDRRQQ }}$ \\
\hline SprE-G6Gln/Glu11Gln/Ser1Thr & Tryp & $\mathrm{SHSQKR}^{-1} / \underline{\mathrm{T}^{1} \text { LLDPQDRRQQ }}$ \\
\hline SprE-G6Gln/Glu11Gln/Ser1Ala & Tryp & $\mathrm{SHSQKR}^{-1} / \mathrm{A}^{1}$ LLDPQDRRQQ \\
\hline SprE-G6Gln/Glu11Gln/Ser1Val & Tryp & $\mathrm{SHSQKR}^{-1} / \underline{\mathrm{V}^{1} \text { LLDPQDRRQQ }}$ \\
\hline \multirow[t]{3}{*}{ SprE-Leu2Val } & Th & SHSQKRS $^{1} / \underline{\mathrm{V}^{2} \mathrm{LDPEDRRQ}}$ \\
\hline & $\operatorname{Tryp}^{\mathrm{b}}$ & $\mathrm{SHSQKR}^{-1} / \underline{\mathrm{S}^{1} \mathrm{VLDPEDRRQ}}$ \\
\hline & & $\mathrm{RRQE}^{11} / \underline{\mathrm{V}^{12} \mathrm{ADTTE}} \mathrm{A}$ \\
\hline
\end{tabular}

${ }^{\text {abc }}$ A mixture of two polypeptides; Detected N-terminal amino acids of the SprE species were underlined.

The substrate specificity of SprE strongly suggested that pro-SprE could not be processed to active SprE as either proor mature SprE unable to cleave GAR-MCA. More interestingly, pro-SprE possessed a faint activity for LLEand LLQ-MCA. These results strongly suggested that, the propeptide shortening via cleavages at the $\mathrm{Glu}^{-15}-\mathrm{Glu}^{-14}$ or $\mathrm{Glu}^{-8}-\mathrm{Ser}^{-7}$ bond observed in the purified pro-SprE (Fig-1 a,b lane 1,2 ) was mediated by pro-SprE, but not by mature SprE and finally maturation achieved by cleaving between $\mathrm{Arg}^{-1}-\mathrm{Ser}^{1}$ in a hetero catalytic manner.

\subsection{Significance of $\mathrm{Ser}^{1}$ and Leu ${ }^{2}$ in the Catalytic Processes}

As $\mathrm{Val}^{1}$ is highly conserved in the mature form of all Staphylococcal glutamyl endopeptidases and is indispensible for enzyme catalysis [26, 29], we examined the requirement of the N-terminal $\operatorname{Ser}^{1}$ on the activity of SprE. Because the wild-type SprE subsequently underwent auto-degradation after trypsin treatment, we used SpreE-Glu6Gln/Glu11Gln instead of wild-type SprE as control and for mutagenesis. When Ser ${ }^{1}$ of SpreE-Glu6Gln/Glu11Gln was mutated to Thr, Ala and Val, the activity of SprE after trypsin treatment was decreased to $55 \%$, $36 \%$, and $31 \%$ respectively (Fig. $4 \mathrm{a}$ ). Thus, the amino acid $\mathrm{Ser}^{1}$ was required at $\mathrm{N}$-terminal position for the maximal activity and amino acids similar to Ser are more preferable, whereas the extent is not so absolute compared to the $\mathrm{Val}^{1}$ requirements of GluV8 [26,29].The substitution of $\mathrm{Leu}^{2}$ to Val also reduced the activity to $44 \%$ of that of wild-type (Fig. $4 \mathrm{~b}$ ).

Altogether, these results indicated the N-terminal amino acids involved with the substrate interaction in the enzyme catalysis.
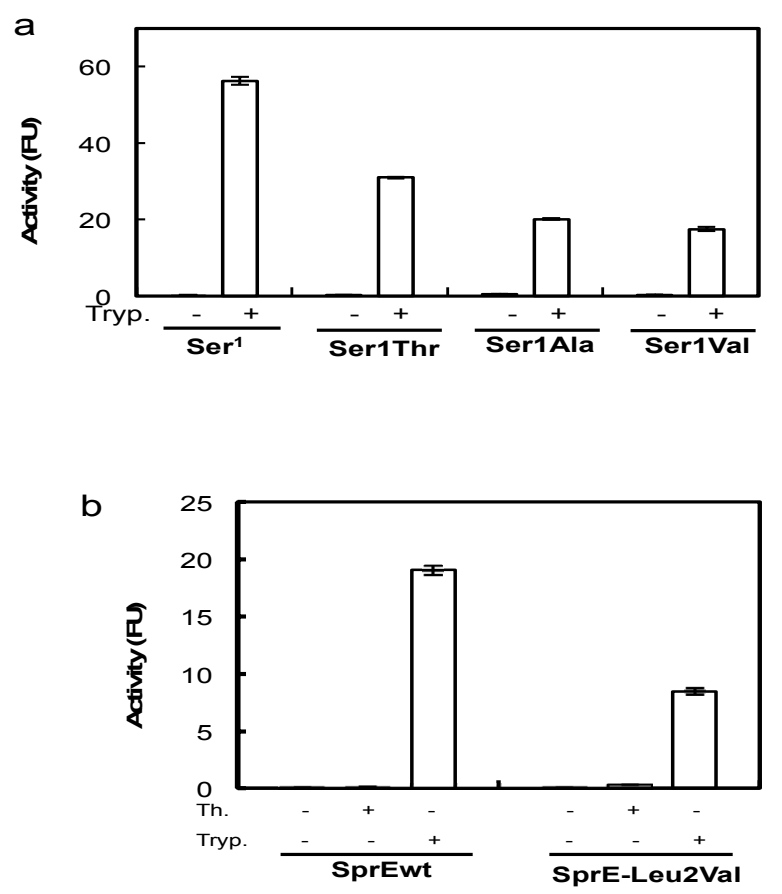

Fig 4. Comparison of proteolytic activities of SprE substitution mutants. (a)Proenzymes of SprE-Glu6Gln/Glu11Gln and it's Ser'substitution derivatives (Ser1Thr, Ser1Ala and Serl Val) $(10 \mu \mathrm{g})$ were incubated at $37^{\circ} \mathrm{C}$ without or with $0.003 \mu \mathrm{g}$ trypsin (Tryp), as described in the materials and methods section. The proteolytic activities (mean $\pm S D ; n=3$ ) of the samples were determined with LLE-MCA. (b)Proenzymes of SprEwt and

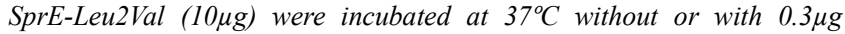
thermolysin (Th) and $0.003 \mu \mathrm{g}$ trypsin (Tryp) for $2 \mathrm{~h}$ and their proteolytic activities (mean $\pm S D ; n=3$ ) were measured as discussed in the materials and methods. 


\section{Discussion}

We purified mixture of two SprE proenzymes starting $\mathrm{Glu}^{-14}$ and $\mathrm{Ser}^{-7}$ when expressed in E. coli and these proenzymes showed trace Glu-specific activity towards LLE- and LLQ-MCA. When all of the three susceptible Glu in the prosequence $\left[\mathrm{Glu}^{-15}, \mathrm{Glu}^{-14}\right.$ and $\mathrm{Glu}^{-8}$ ] to stop auto degradation in the prosequence, then only one proenzymes starting with Lys $^{-2}$ (Lys ${ }^{-2}$ pro-SprE) was purified (data not shown). However, no such cleavage was found when catalytically inert SprE (active site $\mathrm{Ser}^{180}$ was mutated to Ala) was purified from $E$ coli lysate (unpublished data). Taken together, we proposed that full-length pro-SprE truncated it's prosequence to shorter zymogens ( $\mathrm{Glu}^{-14}$ pro-SprE, $\mathrm{Ser}^{-7}$ pro-SprE or Lys ${ }^{-2}$ pro-SprE) by autocatalysis to facilitate final heterocatalytic maturation. Furthermore, absences of any R-X or/S-X cleavage activity by the SprE (both pro-and mature) reiterated the inability of final maturation by autocatalysis.

We have been reported for the first time the in vitro processing of glutamyl endopeptidase proenzymes from Enterococcus faecalis, (pro-SprE) expressed in E.coli. In this study we showed that trypsin (molar ratio 3300:1) could efficiently processed pro-SprE to mature SprE in dose-dependent manner, whereas thermolysin (molar ratio 33:1) could also processed leaving nearly inactive SprE (Fig-1d). Sequencing data confirmed that thermolysin processed between $\mathrm{Ser}^{1}-\mathrm{Leu}^{2}$. Whereas taken together the sequencing data of trypsin processed SprEwt, SprE-Glu11Gln and SprE-Glu6Gln/Glu11Gln, it was confirmed that trypsin processed between $\operatorname{Arg}^{-1}-\operatorname{Ser}^{1}$ (Table 1) leaving fully active SprE, however the wild-type SprE subsequently underwent auto degradation preferentially between $\mathrm{Glu}^{11}-\mathrm{Val}^{12}$ and $\mathrm{Glu}^{6}-\mathrm{Asp}^{7}$ after maturation. $E$. faecalis strain V583 and TX0411 were reported to carries the $h$ tr $A$ gene that produce the trypsin-like serine protease [34,35]. Thus we hypothesize, E. faecalis strains expressing trypsin might efficiently processes secreted pro-SprE zymogens to fully active mature SprE in vivo and might be more pathogenic than strains which do not express trypsin.

It was reported that $\mathrm{N}$-terminal $\mathrm{Val}^{1}$ truncated GluV8 (GluV8 starting with $\mathrm{Ile}^{2}$ ) loose enzyme activity [29]. Moreover, it is known that docking of an $\mathrm{N}$-terminal residue of a chymotrypsin family serine protease affected substrate-binding sites of the enzyme [36]. Thus the length of N-terminal region was indispensible for the glutamyl endopeptidase activity and in absence of trypsin like protease; other thermolysin like protease(s) might processes SprE at $\mathrm{Ser}^{1}-\mathrm{Leu}^{2}$ leaving $\mathrm{Leu}^{2}$ as N-terminal amino-acids that still retained very faint residual geltinase activity.

As the wild-type active SprE rapidly underwent autodegradation at $\mathrm{Glu}^{11}-\mathrm{Val}^{12}$ and Glu ${ }^{6}-\mathrm{Asp}^{7}$ (Table-1), we mutated the susceptible Glu- residues $\left(\mathrm{Glu}^{11}\right.$ and $\left.\mathrm{Glu}^{6}\right)$ to Gln- and found that the recovery of mature SprE following trypsin treatment was greatly enhanced (approximately two-fold increase when Glu ${ }^{11}$ was mutated and about 2.4-fold when both $\mathrm{Glu}^{11}$ and Glu ${ }^{6}$ were mutated) (Fig-2). However we did not find any auto-degradation between
$\mathrm{Glu}^{17}-\mathrm{Ala}^{18}$ that was reported to be the primary auto-degradation site [18]. We supposed that auto degradation first attacked at $\mathrm{Glu}^{11}-\mathrm{Val}^{12}$ and then the enzymetically inactive degraded products underwent further sequential degradation at many points including $\mathrm{Glu}^{17}-\mathrm{Ala}^{18}$.

Staphylococcal GluV8 family proteases were reported to conserve $\mathrm{Val}^{1}$ as $\mathrm{N}$-terminal amino acid and indispensable in enzyme catalysis $[14,25,29]$.To investigate such importance of Ser ${ }^{1}$ of mature SprE, we mutated Ser ${ }^{1}$ to Thr, Ala and Val. In our surprise we found such substitutions were not significantly affect the enzyme activity as in the case of $\mathrm{Val}^{1}$ of GluV8. More over Leu ${ }^{2}$ substitution to Val also reduced Glu-specific activity (SprE-Leu2Val showed 44\% activity of that of the SprEwt) after maturation by trypsin. However, thermolysin again could not raise protease activity even after processing at $\mathrm{Val}^{2}$ position. The possible effects of substitution of N-terminal $\operatorname{Ser}^{1}$ or $\mathrm{Leu}^{2}$ on SprE activity might be due to the difference in structure as well as in hydrophobicity with respect to $\mathrm{Ser}^{1}$ and $\mathrm{Leu}^{2}$. Increase hydrophobicity might bury the $\mathrm{N}$-terminal residue more compactly making less available for substrate interaction to $\mathrm{S} 1$ site. Thus we hypothesized the N-terminal amino acid residue of the glutamyl endoppetidase is evolutionally conserved for facilitating heterocatalytic maturation and to favour substrate interaction to the active site.

In conclusion, we reported in vitro processing of SprE by trypsin, and investigated the significance of $\mathrm{N}$-terminal residue that may enlighten further study on the in vivo processing and pathogenic importance of SprE.

\section{Acknowledgements}

We greatly acknowledge T. Kobayakawa (Nagasaki University) for the technical assistance. This work was supported by grants-in-aid for scientific research from the Ministry of Education, Science, Sports, and Culture of Japan (to Y. O.-N., S. K. and T. K. N.)

\section{Abbreviations}

GluV8 \& SprE, glutamyl endopeptidase from Staphylococcus aureus and Enterococcus faecalis respectively; ac-, acetyl; boc, t-butyloxycarbonyl-[(2S)-2-amino-3-(benzyloxycarbonyl) propionyl]; Z-, benzyloxycarbonyl-; MCA, 4-methylcoumaryl-7-amide, Th, thermolysin; Tryp, trypsin

\section{References}

[1] Murry BE, "The life and times of the enterococcus" ClinMicrobiol Rev, 1990, Vol-3, pp 46-65

[2] Jett BD, Huycke MM, Gilmore MS, "Virulence of Enteroccci”' Clin Microbiol Rev, 1994,Vol 7, pp 472-478

[3] Sedgley CM, Molander A, Flannagan SE, et al, "Phenotype, genotype and virulence of endodontic enterococci" Oral Microbiol Immunol 2005, Vol 20, pp 10- 19 
[4] Stuart CH, Schwartz SA, Beeson TJ, Owatz CB, "Enterococcus faecalis: its role in root canal treatment failure and current concepts in retreatment" J Endod 2006, Vol 32, pp 93-98

[5] Rich RL, Kreikemeyer B, Owens RT, et al, "Ace is a collagen-binding MSCRAMM from Enterococcus faecalis" J Biol Chem, 1999, Vol 274, pp 26939-26945.

[6] Hubble TS, Hatton JF, Nallapareddy SR, Murray BE, Gillespie MJ, "Influence of Enterococcus faecalis proteases and the collagen binding protein, Ace, on adhesion to dentin" Oral Microbiol Immunol, 2003, Vol 18, pp 121-126.

[7] Wang L, Dong M, Zheng J, Song Q, Yin W, Li J and Niu W, "Relationship of biofilm formation and gelE gene expression in Enterococcus faecalis recovered from root canals in patients requiring endodontic retreatment" $J O E$, 2011, Vol 37, pp631-636

[8] Park SY, Kim KM, Lee JH Seo SJ and Lee IH, "Extra cellular gillatinase of Enterococcus faecalis destroys a defense system in insect hemolymph and human serum" Infect Immun, 2007 Vol 75, pp 1861-1869.

[9] Potempa J, and Pike RN, "Corruption of innate immunity by bacterial proteases” J. Innate Immun, 2009 Vol 1, pp 70-87

[10] Schmidtchen A, Frick IM Anderson E, Tapper H and Bjorck $\mathrm{L}$, "Proteinases of common pathogenic bacteria degrade and inactivate the antibacterial peptide LL-37" Mol. Microbiol, 2002, Vol 46, pp 157-168

[11] Qin X, Shing K V, Weinstock GM and Murrray BE. "Effects of Enterococcus faecalis $f s r$ genes on production of gilatinase and a serine protease and virulence" Infect. Immun, 2000, Vol 68, pp 2579-2586.

[12] Gutschik E, Moller S and Christensen N, "Experimental endocarditis in rabbits.3. Significance of the proteolytic capacity of the infecting strain of Enterococcus faecalis". Acta Pathol. Microbiol. Scand, 1979, Vol B87, pp 353-362.

[13] Shing KV, Nallapareddy SR, Nannini EC, and Murray BE, "Fsr-independent production of protease(s) may explain the lack of attenuation of an Enterococcus faecalis $f s r$ mutant vursus a gelE-sprE in induction of endocarditis" Infect. Immun, 2005, Vol 73, pp 4888-4894.

[14] Prasad L, Leduce Y, Hayakawa K and Delbaere L.T. “The structure of a universely employed enzyme:V8 protease from Staphylococcus aureus" 2004, Acta Crystallogr. Sect. D, Vol 60, pp 256-259.

[15] Nemoto $\mathrm{T}$ K, Ono $\mathrm{T}$, Shimoyama $\mathrm{Y}$, Kimura $\mathrm{S}$ and Ohara-Nemoto, Y, "Determination of three amino acids causing alteration of proteolytic activities of Staphylococcal glutamyl endopeptidase" J. Biol. Chem, 2009, Vol 390, pp 277-275.

[16] Engelbert M, Mylonakis E, Ausubel FM, Calderwood SB and Gilmore MS. "Contribution of gilatinase, serine protease, and $f_{s r}$ to the pathogenesis of Enterococcus faecalis endophthalamitis" Infect. Immun, 2004, Vol 72, pp 3628-3633.

[17] Sifri CD, Mylonakis E, Shing KV, Qin X, Garsin DA, Murry BE, Ausubel FM, and Calderwood, SB. "Virulence effects of Enterococcus faecalis protease genes and the quorum-sensing locus $f_{s r}$ in Caenorhabditis elegans and mice" Infect. Immun, 2002, Vol 70, pp5647-5650.
[18] Kawalec M, Potempa J, Moon JL, Travis J and Murray BE, "Molecular diversity of a putative virulence factor: Purification and characterization of an extracellular serine glutamyl endopeptidase of Enterococcus faecalis with different enzymatic activities" J Bacteriol, 2005, Vol 187, pp266-275.

[19] Yoshikawa K, Tsuzuki H, Fujiwara T, Nakamura E, Iwamoto $\mathrm{H}$, Matsumoto $\mathrm{K}$, Shin $\mathrm{M}$, Yoshida $\mathrm{N}$ and Teraoka $\mathrm{H}$, " Purification, Charachterization and gene cloning of a novel glutamic acid-specific endopeptidase from Staphylococcus aureus ATCC12600" Biochim. Biophys. Acta,1992, Vol 1121, pp 221-228.

[20] Ohara-Nemoto Y, Ikeda Y, Kobayashi M, Sasaki M, Tajika S and Kimura S, "Characterization and molecular cloning of a glutamyl endopeptidase from Staphylococcus epidermidis" Microb. Pathog, 2002, Vol 33, pp33-41.

[21] Drapeau GR, "Role of metalloprotease in activation of the precursor of Staphylococcal protease" J bacterial, 1978, Vol 136, pp607-613.

[22] Shaw L, Golonka E, Potempa J and Foster S J, "The role and regulation of the extracellular proteases of Staphylococcus aureus" Microbiology, 2004, Vol 150, pp217-228.

[23] Nickerson NN, Prasad L, Jacob L, Delbaere LT and McGavin MJ, "Activation of the SspA serine protease zymogen of Staphylococcus aureus proceeds through unique variations of a trypsinogen mechanism and is dependent on both auto-catalytic and metallo protease-specific processing" J. Biolo. Chem, 2007, Vol 47, pp34129-34138.

[24] Rouf SMA, Ohara-Nemoto Y, Shimoyama Y, Kimura S, Ono $\mathrm{T}$ and Nemoto, TK, "Propeptide processing and proteolytic activity of proenzymes of the Sthaphylococcal and Enterococcal GluV8-family protease" Indian J Biochem Biophys, 2012,Vol 49(6), pp421-7

[25] Nemoto TK, Ohara-Nemoto Y, Ono T, Kobayakawa T, Shimoyama Y, Kimura S, and Takagi T, "Characterization of the glutamyl endopeptidase from Staphylococcus aureus expressed in Escherichia coli” FEBS.J, 2008, Vol 275, pp573-587.

[26] Ohara-Nemoto Y, Ono T, Shimoyama Y, Kimura S and Nemoto T K, "Homologous and heterologous expression and maturation processing of extracellular glutamyl endopeptidase of Staphylococcus epidermidis" J.Biol. Chem, 2008, Vol 389, pp 1209-1217.

[27] Dubin G, Chmiel D, Mak P, Rakwalska M, Rzychon M and Dubin A, "Molecular cloning and biochemical characterization of proteases from Staphylococcus epidermidis" J.Biol. Chem, 2001, Vol 382, pp 1575-1582.

[28] Yokoi K, Kakikawa M, Kimoto H, Watanabe K, Yasukawa H, Yamakawa A, Taketo A and Kodaira K, "Genetic and Biochemical characterization of glutamyl endopeptidase of Staphylococcus warneri" M Gene, 2001, Vol 281, pp115-122.

[29] Ono T, Ohara-Nemoto Y, Shimoyama Y, Okawara H, Kobayakawa T, Baba T T, Kimura $\mathrm{S}$ and Nemoto $\mathrm{T} \mathrm{K}$. "Amino acid residues modulating the activities of Staphylococcal glutamyl endopeptidases" J. Biol. Chem, 2010, Vol 391, pp1221-1232. 
[30] Ono T, Nemoto TK Shimoyama Y, Kimura S and Ohara-Nemoto Y, “ An Escherichia coli expression system for glutamyl endopeptidases optimized by complete suppression of autodegradation" Analytical Biochemistry. 2008, Vol 381, pp74-80.

[31] Rasband WS., ImageJ, U. S. National Institutes of Health, Bethesda, Maryland, USA, http://imagej.nih.gov/ij/, 1997-2011.

[32] Rice K, Peralta R, Bast D, de Azavedo J and McGavin MJ, "Description of staphylococcus serine protease ( $s s p$ ) operon in Staphylococcus aureus and nonpolar inactivation of $\operatorname{ssp} A$-encoded serine protease" Infect. Immun, 2001, Vol 69, pp159-169.

[33] Qin X, Shing KV, weinstock GM and Murray BE.
"Characterization of $f_{s} r$, a regulator controlling expression of gelatinase and serine protease in Enterococcus faecalis OG1RF”J Bacteriol, 2001, Vol 183,pp3372-3382.

[34] Paulsen I, Banerjei L, Myers G et al, "Role of mobile DNA in the evaluation of venomycin-resistant Enterococcus faecalis" Science, 2003, Vol 299 (5615), pp2071-2074.

[35] Weinstock G, Sodergren E et al. Direct Submission, 2010, Genome sequencing center, Washington university school of medicine, 4444 Forest park, Saint-Louis, MO 63108, USA.

[36] Bode W, and Huber R, "Induction of the bovine trypsinogen-trypsin transition by peptides sequentially similar to the N-terminus of trypsin" FEBS Lett,1976, Vol 68, pp231-236. 